\title{
Tobacco use and nicotine dependence among patients with diabetes and hypertension in Ballabgarh, India
}

\author{
Rakesh Kumar, Shashi Kant, Ankit Chandra, Anand Krishnan \\ Centre for Community Medicine (CCM), All India Institute of Medical Sciences (AIIMS), New Delhi, India
}

\begin{abstract}
Tobacco use is one of the most important modifiable risk factors for cardiovascular mortality and has a synergistic effect with diabetes and hypertension. This study was conducted to estimate the prevalence of tobacco use and nicotine dependence among adult diabetic and/or hypertensive patients. We conducted a crosssectional study among 419 consecutively enrolled patients from the non-communicable diseases (NCDs) outpatient clinic of a secondary level hospital in Ballabgarh, India between July 2018 and January 2019. We administered a pre-tested questionnaire to
\end{abstract}

Correspondence: Dr. Rakesh Kumar, Associate Professor, Centre for Community Medicine (CCM), All India Institute of Medical Sciences (AIIMS), Old OT Block, New Delhi 110029, India.

Tel. +91.011.26593233.

E-mail: dr.rakesh3105@gmail.com

Key words: Tobacco; NCD; smoking, nicotine dependence.

Contributions: All the authors made a substantive intellectual contribution. RK, guarantor. All the authors have read and approved the final version of the manuscript and agreed to be accountable for all aspects of the work.

Conflict of interest: The authors declare that they have no competing interests, and all authors confirm accuracy.

Ethics approval: Ethical clearance was obtained from the Institute Ethics Committee (IEC ref. IEC/396/6/2017).

Funding: This study was part of intramural project of the All India Institute of Medical Sciences (AIIMS), New Delhi (project number A543). The funders have no role in study design, data collection, analysis, result interpretation or manuscript preparation.

Informed consent: Written informed consent was obtained from all study participants.

Received for publication: 9 February 2021.

Accepted for publication: 14 July 2021.

${ }^{\text {CC }}$ Copyright: the Author(s), 2021

Licensee PAGEPress, Italy

Monaldi Archives for Chest Disease 2022; $92: 1799$

doi: 10.4081/monaldi.2021.1799

This article is distributed under the terms of the Creative Commons Attribution Noncommercial License (by-nc 4.0) which permits any noncommercial use, distribution, and reproduction in any medium, provided the original author(s) and source are credited. assess tobacco use and Fagerstrom Test for Nicotine Dependence (FTND) to assess nicotine dependence. Current tobacco users were defined as those who smoked in the past seven days. Nicotine dependence was classified as low, moderate or high for the FTND score of $0-3,4-6$, and 7-10, respectively. Seventy-nine patients had diabetes, 226 had hypertension, and 114 had both. The prevalence of tobacco use was 20.8\% (95\% CI : 17.1 - 24.9); prevalence of smoking was $15 \%(95 \% \mathrm{CI}: 11.9-18.8)$ and smokeless tobacco use was 7.2\% (95\% CI: 5 - 10.1). Moderate to high nicotine dependence was found among $59.7 \%$ of tobacco users; $75.9 \%$ tobacco users attempted to quit tobacco in the past one month. One-fifth of attendees of a NCD clinic in a secondary level hospital used tobacco, most of whom had moderate-to-high nicotine dependence. High level of nicotine dependence and inability to quit despite making an attempt for it necessitates the inclusion of tobacco cessation services in the management of patients with non-communicable diseases.

\section{Introduction}

Cardiovascular diseases (CVDs) are the leading causes of premature mortality in the world. In 2017, CVDs caused 330 million years of life lost, and 35.6 million years lived with disability, and burden was higher in the low-and middle-income countries (LMICs) [1,2]. In 2010-13, CVDs caused $23.3 \%$ of all deaths in India [3].Tobacco use is one major risk factors for CVDs, along with diabetes and hypertension [4] Presence of multiple risk factors have a synergistic effect on morbidity and mortality $[5,6]$. A cohort study found that the hazard ratio for CVD mortality increased to 2.3 (95\% CI: 2.1-2.5) for those who smoked over 20 pack years with hypertension compared to those who never smoked [7]. In a nationally representative case-control study in India found that smoking was associated with an increased risk of mortality from any medical cause among women (risk ratio - 2.0; 99\% CI: -1.8 to 2.3 ) and men (risk ratio $-1.7,99 \%$ CI- 1.6 to 1.8 ). The common cause of death among smokers were tuberculosis and vascular or neoplastic diseases [8]. The World Health Organization (WHO)'s Global Action Plan 2013-2020 and the India's National Action Plan and Monitoring Framework for Prevention and Control of NCDs have set a target to reduce the premature mortality from NCD by $25 \%$ and to reduce the prevalence of tobacco use by $30 \%$ by the year $2025[9,10]$.

The prevalence of tobacco use is estimated to be $20.2 \%$ globally and $23.6 \%$ in India among the general population $[11,12]$. There has been a declining trend in the prevalence of tobacco use in the world and India [12,13]. However, this decline is not fast enough to achieve the target of reducing tobacco use by $30 \%$ by 2025. There is handful of data on the patterns and prevalence of 
tobacco use focused among NCD patients at the national and international level $[1,12]$. A metanalysis in Africa estimated the prevalence of active smoking among patients with diabetes and/or hypertension to be $12.9 \%$ [14]. Studies in India have reported the prevalence of tobacco use among diabetes and/or hypertension patients to be ranging from $11 \%$ to $26.6 \%$ [15-18].

In India, various strategies have been used to reduce tobacco use $[19,20]$. But the prevalence of tobacco use in India remains high $[11,13]$. The reason could be high nicotine dependence, which poses a significant challenge in tobacco cessation [21]. Data on nicotine dependence among smokers with diabetes and hypertension is sparse $[15,16]$. The information on tobacco use and nicotine dependence will help in planning and implementation of the tobacco cessation or control strategy effectively among tobacco users with hypertension and diabetes. Therefore, we conducted this study to estimate the prevalence of tobacco use and nicotine dependence among diabetic and hypertension patients in Ballabgarh, Haryana.

\section{Materials and Methods}

This cross-sectional study was conducted at a secondary level hospital in Ballabgarh block of district Faridabad in Haryana, India. It was a fifty bedded hospital, which served to more than 350,000 outpatients every year and had a specialised NCD clinic. We enrolled 419 consecutive attendees of the NCD clinic, aged $\geq 18$ yrs. Data were collected using a structured pre-tested questionnaire in the local language (Hindi) regarding the socio-demographic details and tobacco use between July 2018 till January 2019. Current tobacco use was defined as either tobacco smoking or the use of smokeless tobacco in the last seven days. Patients who reported current tobacco use were further assessed regarding the product type, frequency, age at initiation of tobacco use, duration of use, and attempt to quit in the last one month. Patients using tobacco in both the forms (smoked and smokeless tobacco) were classified into smokers and smokeless tobacco users based on the predominant use (based on the quantity and the consumption years). We also assessed the current tobacco users for nicotine dependence using revised Fagerstrom Test for Nicotine Dependence (FTND) for smokers [22] and smokeless tobacco users [23]. FTND consists of various items, and their sum produces a score ranging from $0-10$.

Data were entered in Microsoft Excel and was analysed using Stata13. Descriptive analysis was done for the prevalence and pattern of tobacco use. Prevalence of tobacco use was reported as the proportion with the $95 \%$ confidence interval. Nicotine dependence was reported in the category of low, moderate and high. A score of 0-3 was considered as low dependence, 4-6 as moderate dependence and 7-10 as high dependence [24]. We used bivariable and multivariable logistic regression to assess the determinants of tobacco use and, moderate to high nicotine dependence (FTND score $\geq 4$ ). All factors which were potentially significant $(p<0.2)$ in the bivariable analysis were considered for the multivariable logistic regression model. During the multivariable and bivariable analysis, the most predominant form of tobacco was selected for patients who were using tobacco in both forms (smoke and smokeless).

\section{Results}

A total of 419 patients were included in the study; in which 79 patients had diabetes (18.9\%), 226 had hypertension $(53.9 \%)$ and 114 had both $(27.2 \%)$. Majority of the patients were female $(57.5 \%)$, and were in the age group $61-70$ years $(34.6 \%)$ (Table 1$)$.

Table 1. Socio-demographic profile of the patients with diabetes and hypertension in Ballabgarh, India.

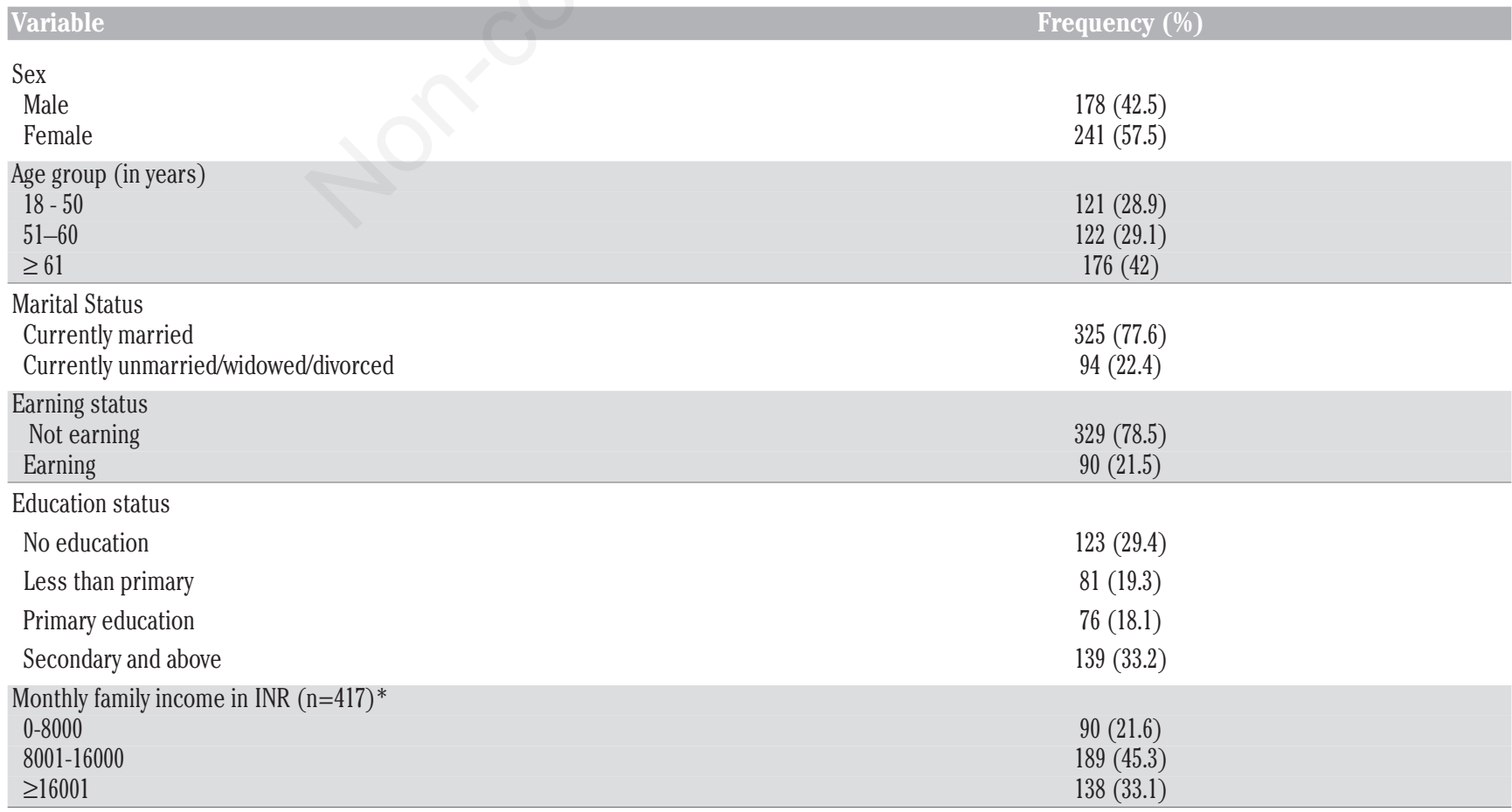

*Two participants refused to provide information on monthly family income. 
The prevalence of tobacco use was 20.8\% ( $\mathrm{n}=87,95 \%$ CI: 17.1 $24.9)$; prevalence of smoking was $15 \%(\mathrm{n}=63,95 \%$ CI: $11.9-$ $18.8)$ and smokeless tobacco use was $7.2 \%(n=30,95 \%$ CI: 5 10.1). Six patients reported using tobacco in both the forms. The prevalence of tobacco use among males and females was $35.4 \%$ (95\% CI: $28.7-42.8)$ and 10\% (95\% CI: $6.7-14.5)$ respectively. The prevalence of tobacco use among diabetic and hypertensive patients was $21.2 \%(n=41,95 \%$ CI: $16-27.6)$ and $20.6 \%(n=70$, 95\% CI: $16.6-25.2)$, respectively.

There were 60 predominant smokers and 27 predominant smokeless tobacco users. Among the patients who predominantly smoked tobacco $(n=60) ; 50$ used bidi, two used cigarettes, six used hookahs, and two used both (hookahs and bidi). The mean age of starting smoking was 30.7 years (SD-14) and mean duration (years of smoking) was 26.3 years (SD-14.3). Among the current predominant smokers, $76.7 \%(\mathrm{n}=46 / 60)$ of the patients reported that they attempted to quit smoking in the last one month. Among the patients who predominantly reported using smokeless tobacco $(n=27)$; four used it with betel leaf, 22 used it as gutka/khaini, one used tobacco in snuff form. The mean age of starting smokeless tobacco use was 34.5 years (SD-12.7) and mean duration (years of using smokeless tobacco) was 21.7 years (SD-15.5). Among the current predominant smokeless tobacco users, $74.1 \%(n=20 / 27)$ of the patients reported that they attempted to quit smokeless tobacco in the last one month.

The mean FTND score among predominant smokers and predominant smokeless tobacco users was 3.6 (SD- 2) and 4.2 (SD1.9), respectively. The proportion of the high level of nicotine dependence among smokeless tobacco users (14.8\%) was more than the smokers $(8.3 \%)$. Most of the tobacco users had moderate nicotine dependence. Moderate to high nicotine dependence was found in $59.7 \%(\mathrm{n}=52)$ of tobacco users $(55 \%$ of predominant smokers and $70.4 \%$ of predominant smokeless tobacco users) (Table 2). In the bivariable regression for determinants of tobacco use; sex, education, marital status and occupation were found potentially significant. In the multivariable regression, sex was significantly associated with tobacco use; males were 5.9 times $(95 \%$ CI: 3-11.6) more likely to be a tobacco user compared to females (Table 3). In bivariable regression for determinants of moderatehigh nicotine dependence (FTND score $\geq 4$ ); tobacco type, quantity per day and age were found to be potentially significant. In the multivariable regression, age was significantly associated with moderate-high nicotine dependence; patients aged 18-59yrs had 2.6 times higher odds (95\% CI: $1.02-6.5)$ of being a moderatehigh nicotine dependence compared to patients aged $\geq 60 \mathrm{yrs}$ (Table 4).

Table 2. Level of nicotine dependence for current predominant smokers $(n=60)$ and current predominant smokeless tobacco users $(n=27)$ among patients with diabetes and hypertension in Ballabgarh, India.

\begin{tabular}{lcc} 
Level of nicotine dependence & Current predominant smokers, n (\%) & Current predominant smokeless tobacco user, n (\%) \\
Low $(0-3)$ & $27(45.0)$ & $8(29.6)$ \\
Moderate (4-6) & $28(46.7)$ & $15(55.6)$ \\
\hline High (7-10) & $5(8.3)$ & $4(14.8)$ \\
\hline
\end{tabular}

Table 3. Determinants of tobacco use among patients with diabetes and hypertension in Ballabgarh, India.

\begin{tabular}{|c|c|c|c|c|c|}
\hline Variables $(n=419)$ & Tobacco users (\%) & Bivariable & lysis & Multivariabl & alysis \\
\hline & & cOR $(95 \% \mathrm{CI})$ & p-value & aOR $(95 \%$ CI) & p-value \\
\hline Sex & & & & & \\
\hline Female $(\mathrm{n}=241)$ & $24(10.0 \%)$ & 1 & & 1 & \\
\hline Male $(n=178)$ & $63(35.4 \%)$ & $4.9(2.9-8.3)$ & $<0.001$ & $5.9(3-11.6)$ & $<0.001$ \\
\hline $\begin{array}{l}\text { Age group (in years) } \\
18-50(\mathrm{n}=121) \\
51-60(\mathrm{n}=122) \\
\geq 61(\mathrm{n}=176)\end{array}$ & $\begin{array}{l}25(20.7 \%) \\
24(19.7 \%) \\
38(21.6 \%)\end{array}$ & $\begin{array}{c}1 \\
0.9(0.5-1.8) \\
1.1(0.6-1.9)\end{array}$ & $\begin{array}{l}0.848 \\
0.847\end{array}$ & Not included & \\
\hline $\begin{array}{l}\text { Education status } \\
\text { No education }(n=123) \\
\text { Less than primary }(n=81) \\
\text { Primary education }(n=76) \\
\text { Secondary and above }(n=139)\end{array}$ & $\begin{array}{l}19(15.4 \%) \\
14(17.3 \%) \\
18(23.7 \%) \\
36(25.6 \%)\end{array}$ & $\begin{array}{c}1 \\
1.21(0.5-2.4) \\
1.7(0.8-3.5) \\
1.9(1.03-3.6)\end{array}$ & $\begin{array}{l}0.728 \\
0.149 \\
0.040\end{array}$ & $\begin{array}{c}1 \\
0.6(0.3-1.5) \\
0.7(0.3-1.7) \\
0.6(0.3-1.2)\end{array}$ & $\begin{array}{c}0.28 \\
0.5 \\
0.15\end{array}$ \\
\hline $\begin{array}{l}\text { Marital status } \\
\text { Currently married }(\mathrm{n}=325) \\
\text { Currently not married }(\mathrm{n}=94)\end{array}$ & $\begin{array}{l}75(23.1 \%) \\
12(12.8 \%)\end{array}$ & $\begin{array}{c}2.1(1.1-3.9) \\
1\end{array}$ & 0.033 & $\begin{array}{c}1.6(0.8-3.2) \\
1\end{array}$ & 0.202 \\
\hline $\begin{array}{l}\text { Family income }(\mathrm{n}=417) \\
0-8000(\mathrm{n}=90) \\
8001-16000(\mathrm{n}=189) \\
\geq 16001(\mathrm{n}=138)\end{array}$ & $\begin{array}{l}20(22.2 \%) \\
35(18.5 \%) \\
31(22.5 \%)\end{array}$ & $\begin{array}{c}1 \\
0.8(0.4-1.5) \\
1.01(0.5-1.9)\end{array}$ & $\begin{array}{l}0.468 \\
0.966\end{array}$ & Not included & \\
\hline $\begin{array}{l}\text { Earning status } \\
\text { Not earning }(\mathrm{n}=329) \\
\text { Earning }(\mathrm{n}=90)\end{array}$ & $\begin{array}{l}58(17.6 \%) \\
29(32.2 \%)\end{array}$ & $\begin{array}{c}1 \\
2.2(1.3-3.8)\end{array}$ & 0.003 & $\begin{array}{c}1 \\
0.97(0.5-1.8)\end{array}$ & 0.942 \\
\hline
\end{tabular}




\section{Discussion}

We found the prevalence of current tobacco use among diabetic and hypertensive patients to be $20.8 \%$. The prevalence of smoking tobacco was higher than the smokeless tobacco use, and male sex was strongly associated with tobacco use. We also found that majority of the tobacco users had moderate to high level of nicotine dependence, and more than one-third of the patients had attempted to quit tobacco use in the past one month.

The observed prevalence of tobacco use was higher among hypertensive patients compared to the studies done in Gujarat (11\% ) [15] and rural Delhi (13.7\%) [17]. The prevalence of tobacco use among diabetic patients was however similar to a study done in Kerala (22.5\%) [16]. The prevalence was less compared to the finding of a study done among middle-class urban participants in India (26.6\%) [18]. All the disparities could be explained due to differences in the study setting and the definitions used for current tobacco users. We found that males sex had higher odds of tobacco use. This finding was in line with a study which analysed the GATS-2 survey data for determinants of tobacco use in India [25]. This can be explained by the social norms in India, where use of tobacco by women is considered a taboo [26,27].

We found that $59.7 \%$ of tobacco users had moderate to high nicotine dependence. Similar findings was reported by a study done in West Bengal (54.5\%) among young adolescents [28]. We found higher nicotine dependence among smokeless tobacco users compared to smokers, which was similar to studies conducted at Puducherry [29], Bhopal [30] and Nepal [31]. Our finding of younger aged patients to be associated with nicotine dependence was similar to a study conducted among male industrial workers [32]. We found a higher proportion of patients who had attempted to quit tobacco in the past one month compared to a national survey $(42 \%)$ conducted among general population [33]. This difference could be due to the study participants, as we had enrolled patients from the NCD clinic who are regularly advised for quitting tobacco or it could be due to social desirability bias.

The high nicotine dependence and inability to quit tobacco among NCD patients suggests need for an effective integration of tobacco cessation services at the NCD clinics under the national programme. Tobacco cessation or control strategy plays a vital role in the management of diabetes and hypertension [34,35]. A treating physician needs to screen for nicotine dependence among NCD patients with tobacco use to provide an appropriate guidance. In the past ten years (2007-2017), there has been an increase of $13 \%$ in the Disability Adjusted Life Years (DALYs) contributed by tobacco use [36]. More accelerated and focused strategies need to be adopted to achieve the target of tobacco reduction by $30 \%$ by the year 2025 $[9,10]$. This study provided an insight over the patterns of tobacco use and dependence on nicotine among the NCD patients, and provides evidence for planning an effective intervention for tobacco cessation. We used a standard tool to assess the nicotine dependence. One of the limitations of the study was the assessment of tobacco use was self-reported by the patients, which could have led to an underestimation of the prevalence of the tobacco due to the social desirability bias. The patients attending NCD clinic may not be representative of all NCD patients in the community. Hence it is recommended that community-based studies may be conducted to generalise these findings and to formulate an effective intervention for tobacco cessation among NCD patients.

Table 4. Factors associated with moderate to high nicotine dependence (FTND score $\geq 4$ ) among patients with diabetes and hypertension in Ballabgarh, India.

\begin{tabular}{|c|c|c|c|c|c|}
\hline \multirow[t]{2}{*}{ Variables $(n=87)$} & \multirow{2}{*}{$\begin{array}{l}\text { Patients with high } \\
\text { nicotine dependence }\end{array}$} & \multicolumn{2}{|c|}{ Bivariable analysis } & \multicolumn{2}{|c|}{ Multivariable analysis } \\
\hline & & cOR $(95 \% \mathrm{CI})$ & p-value & aOR $(95 \% \mathrm{CI})$ & p-value \\
\hline \multicolumn{6}{|l|}{ Predominant tobacco type } \\
\hline Smoke $(\mathrm{n}=60)$ & $33(55.0 \%)$ & 1 & & 1 & \\
\hline Smokeless ( $\mathrm{n}=27)$ & $19(70.4 \%)$ & $1.9(0.7-5.1)$ & 0.180 & $1.8(0.7-4.7)$ & 0.263 \\
\hline \multicolumn{4}{|l|}{ Age of starting tobacco use } & \multirow{3}{*}{\multicolumn{2}{|c|}{ Not included }} \\
\hline$\leq 25$ yrs $(\mathrm{n}=38)$ & $22(57.9 \%)$ & 1 & & & \\
\hline$\geq 26$ yrs $(\mathrm{n}=49)$ & $30(61.2 \%)$ & $1.1(0.5-2.7)$ & 0.753 & & \\
\hline & \multirow{3}{*}{\multicolumn{2}{|c|}{ Not included }} \\
\hline$\leq 20$ yrs $(\mathrm{n}=43)$ & $23(53.5 \%)$ & 1 & & & \\
\hline & $29(65.9 \%)$ & $1.7(0.7-3.4)$ & 0.239 & & \\
\hline \multicolumn{4}{|l|}{ Sex } & \multirow{3}{*}{\multicolumn{2}{|c|}{ Not included }} \\
\hline Female $(n=24)$ & $13(54.2 \%)$ & 1 & & & \\
\hline Male $(n=63)$ & $39(60.0 \%)$ & $1.4(0.5-3.6)$ & 0.511 & & \\
\hline \multicolumn{6}{|l|}{ Age group (in years ) } \\
\hline $18-59(\mathrm{n}=37)$ & $25(67.6 \%)$ & $2.7(1.1-6.7)$ & 0.033 & \multirow{2}{*}{$\begin{array}{c}2.6(1.02-6.5) \\
1\end{array}$} & \multirow[t]{2}{*}{0.045} \\
\hline$\geq 60(\mathrm{n}=50)$ & $27(54.0 \%)$ & 1 & & & \\
\hline \multirow{2}{*}{\multicolumn{4}{|c|}{$\begin{array}{l}\text { Education status } \\
<5^{\text {th }} \text { standard }(n=33)\end{array}$}} & \multirow{3}{*}{\multicolumn{2}{|c|}{ Not included }} \\
\hline & $20(60.6 \%)$ & 1 & & & \\
\hline$\geq 6^{\text {th }}$ standard $(\mathrm{n}=54)$ & $32(59.3 \%)$ & $0.9(0.4-2.2)$ & 0.901 & & \\
\hline \multicolumn{4}{|l|}{ Income status } & \multirow{3}{*}{\multicolumn{2}{|c|}{ Not included }} \\
\hline Not earning $(\mathrm{n}=58)$ & $34(58.6 \%)$ & 1 & & & \\
\hline Earning $(n=29)$ & $18(62.1 \%)$ & $2.7(0.5-2.9)$ & 0.757 & & \\
\hline \multirow{3}{*}{$\begin{array}{l}\text { Monthly family income (INR) } \\
\leq 12000(\mathrm{n}=47) \\
\geq 12001(\mathrm{n}=39)\end{array}$} & & & & \multirow{3}{*}{\multicolumn{2}{|c|}{ Not included }} \\
\hline & $28(59.6 \%)$ & 1 & & & \\
\hline & $23(58.9 \%)$ & $1(0.4-2.3)$ & 0.955 & & \\
\hline
\end{tabular}




\section{Conclusions}

The prevalence of tobacco use among NCD patients was substantial, with more than half of the tobacco users had moderate to high nicotine dependence. More than one-third of the patients had attempted to quit tobacco use in the past one month. There is a need to focus on dealing with the nicotine dependence among tobacco users while planning tobacco cessation strategies.

\section{References}

1. World Health Organization. Noncommunicable diseases country profiles. 2018. Available from: https://apps.who.int/iris/handle/ $10665 / 274512$

2. Kyu HH, Abate D, Abate KH, et al. Global, regional, and national disability-adjusted life-years (DALYs) for 359 diseases and injuries and healthy life expectancy (HALE) for 195 countries and territories, 1990-2017: a systematic analysis for the Global Burden of Disease Study 2017. Lancet 2018;392:1859-922.

3. S Office of the Registrar General \& Census Commissioner [Internet]. Causes of death in India 2010-2013. Accessed: 2019 Apr 30. Available from: http://www.censusindia.gov.in/vital_statistics/causesofdeath.html

4. Hackam DG, Anand SS. Emerging risk factors for atherosclerotic vascular disease: a critical review of the evidence. J Am Med Assoc 2003;290:932-40.

5. Fagard RH. Smoking amplifies cardiovascular risk in patients with hypertension and diabetes. Diabetes Care 2009;32:S429-31.

6. Vidyasagaran AL, Siddiqi K, Kanaan M. Use of smokeless tobacco and risk of cardiovascular disease: A systematic review and meta-analysis. Eur J Prev Cardiol 2016;23:1970-81.

7. Tan J, Zhang X, Wang W, et al. Smoking, blood pressure, and cardiovascular disease mortality in a large cohort of Chinese men with 15 Years follow-up. Int J Environ Res Public Health 2018;15:1-9.

8. Jha P, Jacob B, Gajalakshmi V, et al. A nationally representative case-control study of smoking and death in India. N Engl J Med 2008;358:1137-47.

9. World Health Organization. Global action plan for the prevention and control of NCDs 2013-2020. Accessed: 2019 Apr 20. Available from: http://www.who.int/nmh/events/ncd_action_ plan/en/

10. Government of India, WHO. National action plan and monitoring framework for prevention and control of NCDs. 2012. Accessed: 2019 Jan 20. Available from: http://www.searo.who. int/india/topics/cardiovascular_diseases/National_Action_Plan_ and Monitoring Framework Prevention NCDs.pdf

11. Ministry of Health and Family Welfare, World Health Organization, Tata Institute of Social Sciences. Global Adult Tobacco Survey (GATS2) second round, India, 2016-17.

12. World Health Organization. Global report on trends in prevalence of tobacco smoking 2000-2025. Available from: https://apps. who.int/iris/bitstream/handle/10665/272694/9789241514170eng.pdf

13. Abdulkader RS, Sinha DN, Jeyashree K, et al. Trends in tobacco consumption in India 1987-2016: impact of the World Health Organization Framework Convention on Tobacco Control. Int J f Public Health 2019;64:841-51.

14. Wafeu GS, Tankeu AT, Endomba FTA, et al. Prevalence and associated factors of active smoking among individuals living with hypertension and/or diabetes in Africa: a systematic review and meta-analysis protocol. BMJ Open 2017;7:e015444.

15. Sarkar A, Roy D, Chauhan M, et al. A study on the pattern of selfreported tobacco addiction in hypertensive patients in Gujarat, India. Addict Health 2019;11:35-41.

16. Thresia CU, Thankappan KR, Nichter M. Smoking cessation and diabetes control in Kerala, India: an urgent need for health education. Health Educ Res. 2009;24:839-45.

17. Kishore J, Gupta N, Kohli C, Kumar N. Prevalence of hypertension and determination of its risk factors in rural Delhi. Int $\mathrm{J}$ Hypertens 2016;2016:7962595.

18. Gupta A, Gupta R, Sharma KK, et al. Prevalence of diabetes and cardiovascular risk factors in middle-class urban participants in India. BMJ Open Diabet Res Care 2014;2: e000048.

19. Directorate General of Health Services, Ministry of Health and Family Welfare, Government of India [Internet]. National programme for prevention and control of cancer, diabetes, cardiovascular diseases and stroke. Accessed: 2020 Aug 30. Available from: https://dghs.gov.in/content/1363_3_NationalProgramme PreventionControl.aspx

20. Directorate General of Health Services, Ministry of Health and Family Welfare, Government of India [Internet]. National tobacco control programme. Accessed: 2020 Aug 30. Available from: https://dghs.gov.in/content/1356_3_NationalTobaccoControlPro gramme.aspx

21. Cosci F, Pistelli F, Lazzarini N, Carrozzi L. Nicotine dependence and psychological distress: outcomes and clinical implications in smoking cessation. Psychol Res Behav Manag 2011;4:119-28.

22. Heatherton TF, Kozlowski LT, Frecker RC, Fagerstrom K-O. The Fagerström test for nicotine dependence: a revision of the Fagerstrom Tolerance Questionnaire. Br J Addict 1991;86: 1119-27.

23. Ebbert JO, Patten CA, Schroeder DR. The Fagerström test for nicotine dependence-smokeless tobacco (FTND-ST). Addict Behav 2006;31:1716-21.

24. Mariappan VA, Sahu SK, Sarkar S, et al. Smoked and smokeless tobacco use among pulmonary tuberculosis patients under RNTCP in urban Puducherry, India. Indian J Tubercul 2016;63:158-66.

25. Ruhil R. Sociodemographic determinants of tobacco use in India: Risks of risk factor - an analysis of global adult tobacco survey India 2016-2017. SAGE Open 2019;9:2158244019842447.

26. Morrow M, Barraclough S. Gender equity and tobacco control: bringing masculinity into focus. Global Health Promot 2010;17:2S1-8.

27. World Health Organization. Gender, women, and the tobacco epidemic. World Health Organization, Geneva; 2010.

28. Islam K, Datta AK, Seth S, et al. A study on the prevalence and correlates of nicotine dependence among adolescents of Burdwan Town, West Bengal. Indian J Psychiatry 2019;61: 89-93.

29. Naik BN, Majella MG, Parthibane S, Kar SS. Level of tobacco dependence among tobacco users in an urban slum of Puducherry: A pilot study. J Family Med Prim Care 2017;6:336-9.

30. Pallavi P, Amith HV, Garima B, et al. Assessment of nicotine dependence among the tobacco users in outreach programs: A questionnaire based survey. Int $\mathrm{J}$ Oral Health Med Res 2015;2:34-8.

31. Aryal UR, Bhatta DN, Shrestha N, Gautam A. Assessment of nicotine dependence among smokers in Nepal: a community based cross-sectional study. Tob Induc Dis 2015;13:26-34.

32. Divinakumar KJ, Patra P, Prakash J, Daniel A. Prevalence and 
patterns of tobacco use and nicotine dependence among males industrial workers. Ind Psychiatry J 2017;26:19.

33. Srivastava S, Malhotra S, Harries AD, et al. Correlates of tobacco quit attempts and cessation in the adult population of India: secondary analysis of the Global Adult Tobacco Survey, 2009-10. BMC Public Health 2013;13:263.

34. Whelton PK, Carey RM, Aronow WS, et al. 2017 ACC/AHA/AAPA/ABC/ACPM/AGS/APhA/ASH/ASPC/NMA /PCNA guideline for the prevention, detection, evaluation, and management of high blood pressure in adults: a report of the
American College of Cardiology/American Heart Association Task Force on Clinical Practice Guidelines. J Am Coll Cardiol 2018;71:2199-269.

35. American Diabetes Association. 5. Facilitating behavior change and well-being to improve health outcomes: standards of medical care in diabetes - 2020. Diabetes Care 2020;43:S48-65.

36. Institute for Health Metrics and Evaluation [Internet]. Global burden of diseases. Country profile - India 2015. Accessed: 2020 Apr 16. Available from: http://www.healthdata.org/india 